\title{
STUDY OF THE ${ }^{18} \mathrm{O}+{ }^{64} \mathrm{Ni}$ TWO-NEUTRON TRANSFER REACTION AT $84 \mathrm{MeV}$ BY MAGNEX*
}

\author{
G. Santagati ${ }^{\mathrm{a}}$, B. Paes ${ }^{\mathrm{b}}$, R. Magana Vsevolodovna ${ }^{\mathrm{c}, \mathrm{d}}$

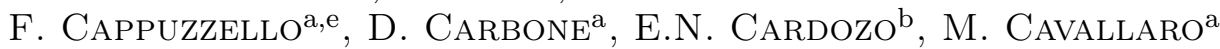 \\ H. Garcìa-Tecocontzi ${ }^{\mathrm{c}}, \mathrm{A} \cdot$ Gargano $^{\mathrm{f}}$, J.L. Ferreira ${ }^{\mathrm{b}}$

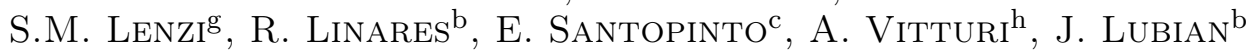 \\ ${ }^{a}$ INFN, Laboratori Nazionali del Sud, 95125, Catania, Italy \\ ${ }^{b}$ Instituto de Fìsica, Universidade Federal Fluminense \\ 24210-340, Niterói, Rio de Janeiro, Brazil \\ ${ }^{\mathrm{c}}$ INFN - Sezione di Genova, 16146 Genova, Italy \\ ${ }^{\mathrm{d}}$ Dipartimento di Fisica e Astronomia, Università di Catania \\ 95125, Catania, Italy \\ eDipartimento di Fisica, Università di Genova, 16164 Genova, Italy \\ ${ }^{\mathrm{f}}$ INFN — Sezione di Napoli, 80126 Napoli, Italy \\ IINFN — Sezione di Padova, 35131 Padova, Italy \\ ${ }^{\mathrm{h}}$ Dipartimento di Fisica e Astronomia Galileo Galilei, Università di Padova \\ 35131 Padova, Italy
}

\section{(Received January 8, 2018)}

A study of the two-neutron transfer reaction of the ${ }^{18} \mathrm{O}+{ }^{64} \mathrm{Ni}$ system at $84 \mathrm{MeV}$ incident energy to the ground and first $2^{+}$excited state of the residual ${ }^{66} \mathrm{Ni}$ nucleus is presented. The experiment was performed at the INFN-LNS (Italy) by using the large acceptance MAGNEX spectrometer. Theoretical models are used in order to disentangle the competition between long-range and short-range correlations.

DOI:10.5506/APhysPolB.49.381

\section{Introduction}

Pairing correlations in nuclei can be probed by two-nucleon transfer reactions [1-11]. Moreover, these reactions can reveal relevant information on more complex competing reactions such as the double charge exchange ones, which are of interest for applications in neutrino-less double beta decay research $[12,13]$. Two-neutron transfer reaction mechanism can proceed either

* Presented at the XXXV Mazurian Lakes Conference on Physics, Piaski, Poland, September 3-9, 2017. 
by the two-neutron transfer in one step, under strong influence of pairing correlations, or in two sequential steps of single neutron transfer (two-step). In the first case, the states of the residual nucleus are fed directly from the ground state of the target. However, here we refer to one-step mechanism even when intermediate transitions through inelastic excitation of the projectile or target are involved [5, 14].

Calculations for the two-neutron transfer reaction $\left({ }^{18} \mathrm{O},{ }^{16} \mathrm{O}\right)$ on various target nuclei were performed in several previous works [14-17]. Full quantum-mechanical calculations for the two-neutron transfer cross section of the ${ }^{58,60,62,64} \mathrm{Ni}\left({ }^{18} \mathrm{O},{ }^{16} \mathrm{O}\right)$ reactions using the distorted wave Born approximation (DWBA) were performed by Kahana and Baltz [18, 19].

A high precision measurement of the ${ }^{64} \mathrm{Ni}\left({ }^{18} \mathrm{O},{ }^{16} \mathrm{O}\right){ }^{66} \mathrm{Ni}$ reaction to the ground and to the first excited state of the residual ${ }^{66} \mathrm{Ni}$ nucleus in order to study the details of the two-neutron reaction on a target in this mass region is presented here.

For the direct two-neutron transfer reaction (or one-step mechanism), CRC calculations were performed. For the sequential transfer (or two-step mechanism), two-step distorted wave Born approximation (DWBA) calculations were performed in post prior representation.

Different nuclear structure models are used to study the structure of ${ }^{64,66} \mathrm{Ni}$ : the shell model, the microscopic interacting boson model (IBM-2) [20] (using the extended mapping procedure suggested by Otsuka, Arima, and Iachello [21]) and the interacting boson fermion model (IBFM-2) [22].

\section{Experiment and results}

A $113 \mu \mathrm{g} / \mathrm{cm}^{2}$ self supporting ${ }^{64} \mathrm{Ni}$ target was bombarded by a $84 \mathrm{MeV}$ incident energy beam of ${ }^{18} \mathrm{O}^{6+}$ delivered by the Tandem Van der Graaff accelerator of the INFN-LNS laboratory in Catania. The evaluation of the background in the spectrum (due to carbon impurities in the target) was realized performing supplementary runs bombarding a $49 \mu \mathrm{g} / \mathrm{cm}^{2}$ self supporting ${ }^{12} \mathrm{C}$ target with the same beam at the same incident energy.

An overall angular interval of $3^{\circ}<\theta_{\text {lab }}<31^{\circ}$ in the laboratory system was spanned. The outgoing ejectiles produced in the different reactions were momentum analyzed by the MAGNEX spectrometer [17, 23] and detected by the focal plane detector FPD [24] which provided particle identification [25] and trajectories reconstruction [26].

A typical energy spectrum, obtained by applying the ray-reconstruction technique to the experimental data, is shown in Fig. 1. The ${ }^{66} \mathrm{Ni}$ excitation energy $E^{*}=Q_{0}-Q$ is represented in the energy axis, where $Q_{0}$ is the $Q$-value $(2.862 \mathrm{MeV})$ for the g.s. to g.s. transition. 


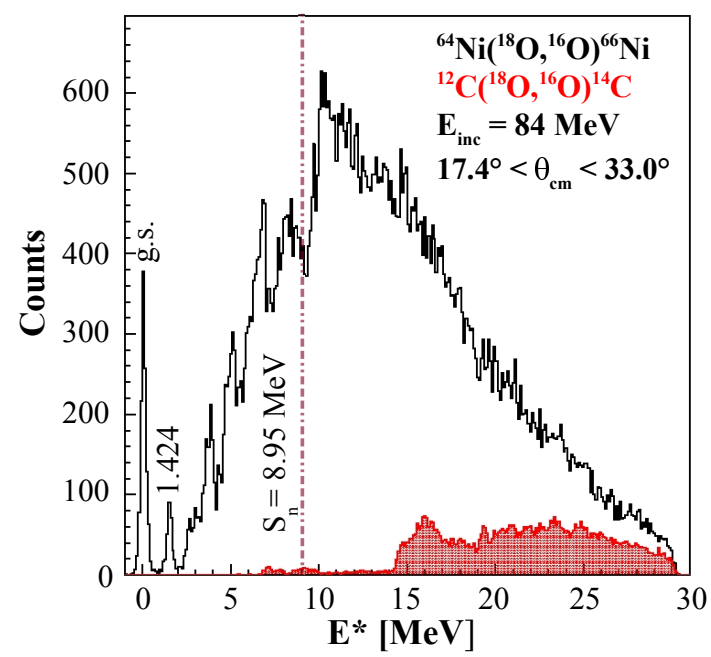

Fig. 1. (Color online) Excitation energy spectrum of ${ }^{66} \mathrm{Ni}$ (black color) obtained from the ${ }^{18} \mathrm{O}+{ }^{64} \mathrm{Ni}$ reaction at $84 \mathrm{MeV}$ and $17.4^{\circ}<\theta_{\mathrm{cm}}<33.0^{\circ}$ for the g.s. transition. The gray/red hatched area corresponds to the background that comes from ${ }^{12} \mathrm{C}$ impurities in the target. A line corresponding to the one-neutron separation energy $\left(S_{n}\right)$ is also indicated.

The particular shape of this spectrum can be explained considering the high level density of ${ }^{66} \mathrm{Ni}$ [27], the kinematic matching conditions which give a maximum cross section at $E_{\mathrm{opt}}^{*}=12.2 \mathrm{MeV}$ and $L_{\mathrm{opt}}=5 \hbar$ [28], and the three-body kinematics related to the one-neutron emission. The large bump may be associated to the competition between the two-neutron transfer mechanism and dissipative phenomena [29].

In Fig. 2, the experimental angular distributions for the transfer to the g.s. and to the first excited state of ${ }^{66} \mathrm{Ni}$ are shown. The angular resolution is $0.3^{\circ}$. The error bars correspond to the uncertainty on the solid angle determination, the statistical error and the background subtraction. A scale error of $10 \%$ in the cross section, coming from the uncertainties of the target thickness and beam integration by the Faraday cup, is common to all the angular distibution points and is not included in the error bars.

The angular distribution of the elastic transfer is bell-shaped near the grazing angle, and at forward angles, the cross section oscillates. This phenomenon, observed in heavy-ion reactions, is a consequence of diffraction between near-side and far-side components of the reaction flux, signaling also an interesting transparency of the ${ }^{64} \mathrm{Ni}$ nucleus for the far-side wave. The forward angle oscillations were not observed in the experimental data of Ref. [19] since not only the authors were not able to measure at forward angles, but in addition, due to the limited angular distribution. 

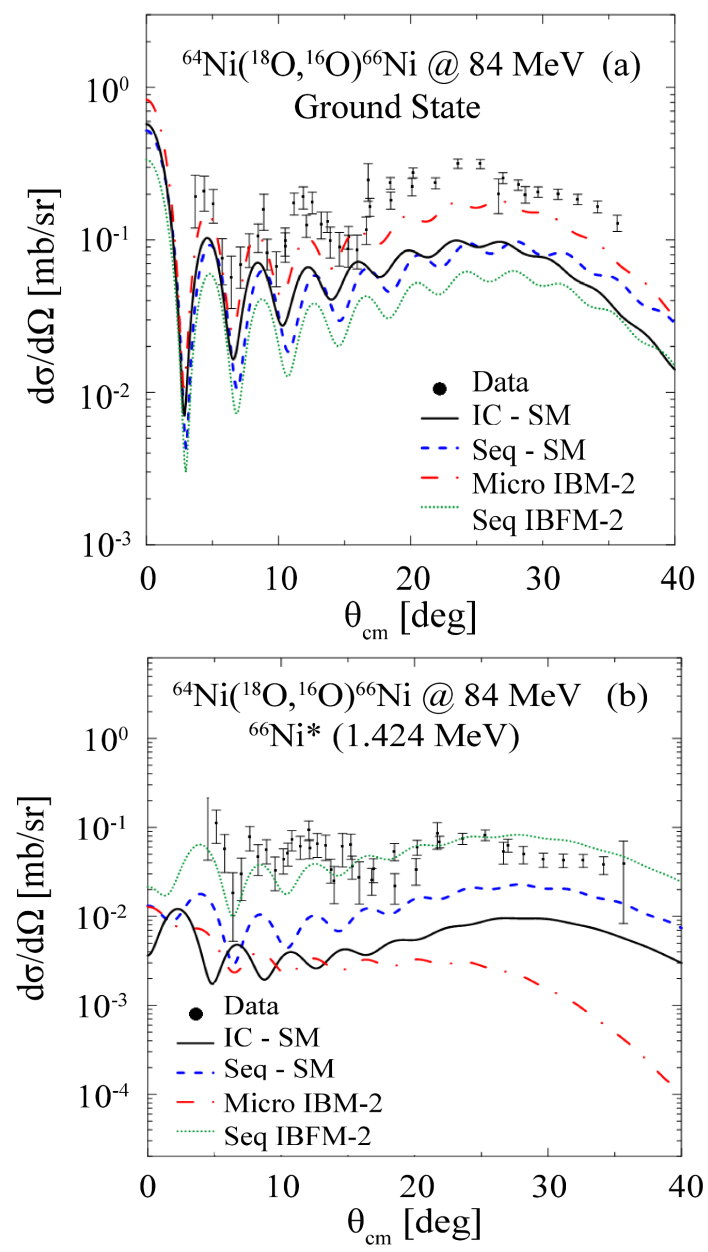

Fig. 2. Experimental angular distributions for the ground state (a) and $1.424 \mathrm{MeV}$ (b) of ${ }^{66} \mathrm{Ni}$ compared with calculations.

\section{Calculations and results}

Two different models were used for the calculation of the two-neutron transfer reaction.

The first one assumes that the transfer reaction of the two neutrons occurs simultaneously, or in a direct way. This is the so-called independent coordinate model, where the relative movement between the two particles is taken into account and all the quantum numbers of the individual neutrons are considered during the transfer process. The description of this approach can be found in Ref. [30]. 
The second one considers that the transfer of the two neutrons occurs in two steps, passing through an intermediate partition, known as a sequential or two-step mechanism. To perform microscopic-independent coordinates and sequential calculations, it is necessary to determine the spectroscopic amplitude for each target and projectile overlap. Two possibilities have been explored for this medium-mass system, the shell model (SM) and the IBM method. The obtained results are compared with the experimental data in Fig. 2.

Spectroscopic amplitudes used in the one-step mechanism (direct) and in two-step mechanism (sequential) are taken from Shell Model calculations using the model space bjuff with the effective phenomenological interaction of Ref. [31] and the microscopic IBM-2 method [20] for the one-step process, and the IBFM-2 method [22] for the sequential process.

\section{Conclusions}

The ${ }^{66} \mathrm{Ni}$ excitation energy spectrum up to about $30 \mathrm{MeV}$ and high quality angular distributions for the ${ }^{64} \mathrm{Ni}\left({ }^{18} \mathrm{O},{ }^{16} \mathrm{O}\right){ }^{66} \mathrm{Ni}$ transfer reactions were measured.

For the two-neutron transfer reactions to the ground state of the residual nucleus ${ }^{66} \mathrm{Ni}$, our CRC cross-section analysis suggests that short-range pairing correlations play an important role (more evident with IBM-2 and IBFM-2).

In the two-neutron transfer reaction to the first excited state of ${ }^{66} \mathrm{Ni}$, where the collectivity is known to be important, the predominance of the two-step reaction mechanism was verified. This confirms the long-range effect of the wave function of this state.

Both conclusions are independent on the nuclear structure model used.

\section{REFERENCES}

[1] M. Cavallaro et al., Phys. Rev. C 93, 064323 (2016).

[2] D. Carbone et al., J. Phys.: Conf. Ser. 312, 082016 (2011).

[3] T. Takemasa, H. Yoshida, Nucl. Phys. A 304, 229 (1978).

[4] P.D. Bond et al., Phys. Rev. C 16, 177 (1977).

[5] M.C. Lemaire, K.S. Low, Phys. Rev. C 16, 183 (1977).

[6] G. Potel et al., Rep. Prog. Phys. 76, 106301 (2013).

[7] I. Inci, A. Vitturi, J. Phys.: Conf. Ser. 321, 012004 (2011).

[8] T. Kammuri, Nucl. Phys. A 259, 343 (1976).

[9] G. Potel et al., Phys. Rev. Lett. 100, 192502 (2008).

[10] M. Cavallaro et al., Phys. Rev. C 88, 054601 (2013). 
[11] F. Cappuzzello et al., Nature Commun. 6, 6742 (2015).

[12] F. Cappuzzello et al., Eur. Phys. J. A 51, 145 (2015).

[13] F. Cappuzzello et al., J. Phys.: Conf. Ser. 630, 012018 (2015).

[14] M. Ermamatov et al., Phys. Rev. C 94, 024610 (2016).

[15] M. Ermamatov et al., Phys. Rev. C 96, 044603 (2017).

[16] D. Carbone et al., Phys. Rev. C 95, 034603 (2017).

[17] F. Cappuzzello et al., Eur. Phys. J. A 52, 167 (2016).

[18] S. Kahana, A.J. Baltz, Advances in Nuclear Physics, vol. 9, Springer, 1977.

[19] E.H. Auerbach et al., Phys. Rev. Lett. 30, 1078 (1973).

[20] J. Barea, F. Iachello, Phys. Rev. C 79, 044301 (2009).

[21] A. Arima, T. Otsuka, F. Iachello, I. Talmi, Phys. Lett. B 66, 205 (1977).

[22] F. Iachello, P.V. Isacker, The Interacting Boson-Fermion Model, Cambridge University, 1991.

[23] A. Cunsolo et al., Eur. Phys. J. ST 150, 343 (2007).

[24] M. Cavallaro et al., Eur. Phys. J. A 48, 59 (2012).

[25] F. Cappuzzello et al., Nucl. Instrum. Methods Phys. Res. A 763, 314 (2014).

[26] D. Carbone, Eur. Phys. J. Plus 130, 143 (2015).

[27] O. Scholten, Z.R. Yu, Phys. Lett. B 161, 13 (1985).

[28] D.M. Brink, Semi-classical Methods for Nucleus-Nucleus Scattering, Cambridge University Press, Cambridge, England, 1985.

[29] M. Cavallaro et al., J. Phys.: Conf. Ser. 515, 012003 (2014).

[30] I.J. Thompson, Comput. Phys. Rep. 7, 167 (1988).

[31] L. Coraggio et al., Phys. Rev. C 89, 024319 (2014). 